\title{
Bayesian Parametric Analysis for Reliability Study of Locomotive Wheels
}

\author{
Jing Lin, PhD, Luleå University of Technology \\ Matthias Asplund, Luleå University of Technology \\ Aditya Parida, PhD, Luleå University of Technology
}

Key Words: Reliability analysis; Bayesian analysis; Locomotive; train wheels; Markov Chain Monte Carlo

\section{SUMMARY \& CONCLUSIONS}

This paper proposes a new approach to study reliability of locomotive wheels with Bayesian framework, utilizing locomotive wheel degradation data sets that can be small or incomplete. In our study, a linear degradation path is assumed and locomotive wheels' installation positions are considered as covariates. A Markov Chain Monte Carlo (MCMC) computational method is also implemented. In the case study, data were collected from a Swedish railway company. This data includes, the diameter measurements of the locomotive wheels, total distances corresponding to their "time to maintenance", and the wheels' bill of material (BOM) data. During this study, likelihood functions were constructed for Expontional regression models, Weibull regression models, and lognormal regression models. The results show that the locomotive wheels' lifetimes are dependent on installation positions. For the studied locomotive wheels data, the Lognormal regression model is a better choice, because the model obtained the lowest Deviance Information Criterion (DIC) values. In addition, under current operation situation (e.g. topography) and current maintenance strategies (reprofiled, lubrication, etc.), the locomotive wheels installed in the second bogie have longer lifetimes than those installed in the first bogie; the wheels installed on the "back" axle have longer lifetimes than those on the "front" axle; and the right side wheels' lifetime is shorter than that for the left side under a given running situation.

\section{INTRODUCTION}

The service life of a railroad wheel can be significantly reduced due to failure or damage, leading to excessive cost and accelerated deterioration. Damage data show that a major proportion of wheel damage stems from degradation.

In order to monitor the performance of wheels and make replacement before adverse effects occur, the railway industry uses both preventive and predictive maintenance. [1-5] By predicting train wheel wear, fatigue, tribological aspects, and failures, the railway industry can formulate different preventive maintenance strategies under different time periods. [6] For predictive maintenance, wheel condition monitoring data have been studied to increase the lifetime by knowing the condition of the wheel profile. [7-9] A large number of related studies have been published in the last decade.

One common preventive maintenance strategy (that is used in the case study) is wheel re-profiling after running a certain distance, with its diameter being measured; if it reduces to a pre-specified diameter, the wheel will be replaced by a new one. In order to optimize such maintenance strategies, some researchers started looking into wheel degradation utilization data to determine reliability and failure distribution. [10] However, these studies cannot solve the combined problem of small data samples and incomplete data sets while simultaneously considering the influence of several covariates. For example, to avoid the potential influence of the different locations of wheels, Freitas [10] only consider those on the left side of axle number and on certain specified cars. Yang and Letourneau [5] suggest that certain attributes, including a wheel's installed position (right or left), might influence its wear rate, but they do not provide case studies.

To address the above issues, this paper undertakes a reliability study using a Bayesian survival analysis framework to explore the impact of the wheel's installed position on its service lifetime and to predict its reliability characteristics. [11] In section 2, the Expontional regression models, Weibull regression models, and lognormal regression models are used to establish the lifetime of locomotive wheels using degradation data and taking into account the position of the wheel. This position is described by three different discrete covariates: the bogie, the axel and the side of the locomotive where the wheel is mounted. In section 3, a linear degradation path is considered and the case study is performed using Markov Chain Monte Carlo methods. And finally, section 4 offers conclusions and comments.

\section{BAYESIAN PARAMETRIC MODELS}

In reliability analysis, the lifetime data set is usually incomplete, which means only a portion of the subsystem failures (i.e. wheels) are known. For other subsystems, the lifetimes are only known to exceed certain values. Take the locomotive wheels' degradation data for example. If the degradation data is less than the pre-specified diameter, the 
corresponding predicted lifetime is viewed as right-censored. The reason is that under a linear degradation path assumption, we just know those wheels' real lifetime will exceed the predicted lifetime.

Right-censored data are often called Type I censoring in the literature; the corresponding likelihood construction problem has been extensively studied. [12, 13] Suppose there are $n$ individuals whose lifetimes and censoring times are independent. The ith individual has life time $T_{i}$ and censoring time $L_{i}$. The $T_{i} \mathrm{~s}$ are assumed to have probability density function $f(t)$ and reliability function $R(t)$. The exact lifetime $T_{i}$ of an individual will be observed only if $T_{i} \leq L_{i}$. The lifetime data involving right censoring can be conveniently represented by $n$ pairs of random variables $\left(t_{i}, v_{i}\right)$, where $t_{i}=\min \left(T_{i}, L_{i}\right) \quad$ and $\quad v_{i}=1$ if $T_{i} \leq L_{i}$, and $v_{i}=0$ if $T_{i}>L_{i}$. That is, $v_{i}$ indicates whether the lifetime $T_{i}$ is censored or not. The likelihood function is deduced as $[12,13]$

$$
L(t)=\prod_{i=1}^{n}\left[f\left(t_{i}\right)\right]^{v_{i}} R\left(t_{i}\right)^{1-v_{i}}
$$

\subsection{Exponential regression model}

Suppose the lifetimes $\mathbf{t}=\left(t_{1}, \cdots t_{n}\right)$ for $n$ wheels are independent and identically distributed (iid), and the distribution being exponential distribution with a failure rate $\lambda$, where $\lambda>0$. Therefore, the probability density function (pdf) is $f\left(t_{i} \mid \lambda\right)=\lambda \exp \left(-\lambda t_{i}\right)$, the cumulative distribution function (cdf) is $F\left(t_{i} \mid \lambda\right)=1-\exp \left(-\lambda t_{i}\right)$ and the reliability function is $R\left(t_{i} \mid \lambda\right)=1-R\left(t_{i} \mid \lambda\right)$. The incomplete indicators are denoted $\boldsymbol{v}=\left(v_{1}, v_{2}, \cdots, v_{n}\right)$ and the observed data set for the current study is $D_{0}=(n, \mathbf{t}, \mathbf{v})$. From equation (1), the likelihood function of $\lambda$ is

$$
L\left(\lambda \mid D_{0}\right)=\prod_{i=1}^{n}\left(\lambda \exp \left(-\lambda t_{i}\right)\right)^{v_{i}}\left(\exp \left(-\lambda t_{i}\right)\right)^{1-v_{i}}
$$

The $p \times 1$ vector of covariates for the $i$ th wheel is denoted $\mathbf{x}_{\mathbf{i}}=\left(x_{1 i}, \cdots x_{p i}\right)^{\prime}$. Similarly, $\boldsymbol{\beta}$ is a $p \times 1$ vector of regression coefficients, which represents the degree of influences of covariates. Let $\lambda_{i}=\exp \left(\mathbf{x}_{i}^{\prime} \boldsymbol{\beta}\right)$ and the observed data set for current study is denoted by $D=(n, \mathbf{t}, \mathbf{X}, \mathbf{v})$. The likelihood function for the regression coefficients is given by

$$
L(\boldsymbol{\beta} \mid D)=\exp \left(\sum_{i=1}^{n} v_{i} \mathbf{x}_{\mathbf{i}}^{\prime} \boldsymbol{\beta}\right) \exp \left(-\sum_{i=1}^{n} \exp \left(\mathbf{x}_{\mathbf{i}}^{\prime} \boldsymbol{\beta}\right) t_{i}\right)
$$

The prior distributions should be realistic and computationally feasible. There are two common choices for $\boldsymbol{\beta}$ 's prior distributions. [11] One is uniform improper prior distribution, for example, $\pi(\boldsymbol{\beta}) \propto 1$.The other is the normal distribution. As proved by Ibrahim [11], it's a log-concave prior and such kind of choice will be convenient for posterior's computation. To implement the MCMC simulation more easily, a multinomial prior $\boldsymbol{\beta} \sim N_{p}\left(\boldsymbol{\mu}_{\mathbf{0}}, \boldsymbol{\Sigma}_{\mathbf{0}}\right)$ with mean $\boldsymbol{\mu}_{\mathbf{0}}$ and covariance matrix $\Sigma_{\mathbf{0}}$ is assumed. Let $\pi(\cdot)$ denote the prior or posterior distributions for the parameters. The posterior distribution, $\pi(\boldsymbol{\beta} \mid D)$, can be written as

$$
\begin{aligned}
& \pi(\boldsymbol{\beta} \mid D) \propto L(\boldsymbol{\beta} \mid D) \times \pi\left(\boldsymbol{\beta} \mid \boldsymbol{\mu}_{\mathbf{0}}, \boldsymbol{\Sigma}_{\mathbf{0}}\right) \\
& \propto \exp \left(\sum_{i=1}^{n} v_{i} \mathbf{x}_{\mathbf{i}}^{\prime} \boldsymbol{\beta}-\sum_{i=1}^{n} \exp \left(\mathbf{x}_{\mathbf{i}}^{\prime} \boldsymbol{\beta}\right) t_{i}-\frac{1}{2}\left(\boldsymbol{\beta}-\boldsymbol{\mu}_{\mathbf{0}}\right)^{\prime} \boldsymbol{\Sigma}_{\mathbf{0}}^{-\mathbf{1}}\left(\boldsymbol{\beta}-\boldsymbol{\mu}_{\mathbf{0}}\right)\right)
\end{aligned}
$$

It is not easy to get the exact integration results for $\pi(\boldsymbol{\beta} \mid D)$ due to its complexity. Therefore, we select the MCMC method, which has been widely applied to Bayesian statistics since 1990 s, to carry out the posterior inference.

\subsection{Weibull regression model}

Suppose, the lifetimes $\mathbf{t}=\left(t_{1}, \cdots t_{n}\right)$ for $n$ individuals are iid, and the distribution is Weibull, $W(\alpha, \gamma)$, where $\alpha>0$ and $\gamma>0$. The pdf is $f\left(t_{i} \mid \alpha, \gamma\right)=\alpha \gamma t_{i}^{\alpha-1} \exp \left(-t_{i}^{\alpha}\right)$ while the cdf is $F\left(t_{i} \mid \alpha, \gamma\right)=1-\exp \left(-\gamma t_{i}^{\alpha}\right)$ and the reliability function $R\left(t_{i} \mid \alpha, \gamma\right)=1-F\left(t_{i} \mid \alpha, \gamma\right)$. To facilitate the analysis, let $\xi=\ln (\gamma)$, permitting the following representation:

$$
f\left(t_{i} \mid \alpha, \xi\right)=\alpha t_{i}^{\alpha-1} \exp \left(\xi-\exp (\xi) t_{i}^{\alpha}\right)
$$

Similarly, we can get $F\left(t_{i} \mid \alpha, \xi\right)$ and $R\left(t_{i} \mid \alpha, \xi\right)$.

From equation (1), the joint likelihood function for $\alpha$ and $\xi$ is

$$
\begin{aligned}
& L\left(\alpha, \xi \mid D_{0}\right)=\alpha^{\sum_{i=1}^{n} v_{i}} \\
& \times \exp \left(\sum_{i=1}^{n} v_{i} \xi+\sum_{i=1}^{n} v_{i}(\alpha-1) \ln \left(t_{i}\right)-\exp (\xi) t_{i}^{\alpha}\right)
\end{aligned}
$$

To construct the Weibull Regression Model, covariates are introduced through $\xi$. With $\xi_{i}=\mathbf{x}_{\mathbf{i}}^{\prime} \boldsymbol{\beta}$, the likelihood function is given by

$$
\begin{aligned}
& L(\alpha, \boldsymbol{\beta} \mid D)=\alpha^{\sum_{i=1}^{n} v_{i}} \\
& \times \exp \left(\sum_{i=1}^{n} v_{i}\left(\mathbf{x}_{\mathbf{i}}^{\prime} \boldsymbol{\beta}+v_{i}(\alpha-1) \ln \left(t_{i}\right)-\exp \left(\mathbf{x}_{\mathbf{i}}^{\prime} \boldsymbol{\beta}\right) t_{i}^{\alpha}\right)\right)
\end{aligned}
$$

In this paper, it is assumed that $\alpha$ and $\xi$ are independent. Furthermore, it is assumed that the prior distribution of $\alpha$ is a gamma distribution, denoted by $G\left(a_{0}, b_{0}\right)$ The prior distribution can be written as $\pi\left(\alpha \mid a_{0}, b_{0}\right) \propto \alpha^{a_{0}-1} \exp \left(-b_{0} \alpha\right)$. Then, the posterior distribution of $\alpha$ and $\boldsymbol{\beta}$ is:

$$
\begin{aligned}
\pi(\alpha, \boldsymbol{\beta} \mid D) \propto \alpha^{a_{0}-1+\sum_{i=1}^{n} v_{i}} \\
\quad \times \exp \left(\sum_{i=1}^{n}\left(v_{i} \mathbf{x}_{\mathbf{i}}^{\prime} \boldsymbol{\beta}+v_{i}(\alpha-1) \ln \left(t_{i}\right)-\exp \left(\mathbf{x}_{\mathbf{i}}^{\prime} \boldsymbol{\beta}\right) t_{i}^{\alpha}\right)\right. \\
\left.\quad-b_{0} \alpha-\frac{1}{2}\left(\boldsymbol{\beta}-\boldsymbol{\mu}_{\mathbf{0}}\right)^{\prime} \Sigma_{\mathbf{0}}^{-1}\left(\boldsymbol{\beta}-\boldsymbol{\mu}_{\mathbf{0}}\right)\right)
\end{aligned}
$$

\subsection{Lognormal regression model}

Suppose the lifetimes $\mathbf{t}=\left(t_{1}, \cdots t_{n}\right)$ of $n$ wheels are iid, with $\ln (t)$ being normally distributed according to $N\left(\mu, \sigma^{2}\right)$. This implies that $t_{i}$ is lognormally distributed with parameters $\mu$ and $\sigma^{2}$, denoted by $L N\left(\mu, \sigma^{2}\right)$. The pdf and reliability functions for $t_{i}$ are

$$
\begin{aligned}
& f\left(t_{i} \mid \mu, \sigma^{2}\right)=\frac{1}{\sqrt{2 \pi} \sigma_{i}} \exp \left(-\frac{1}{2 \sigma^{2}}\left(\ln \left(t_{i}\right)-\mu\right)^{2}\right) \\
& R\left(t_{i} \mid \mu, \sigma^{2}\right)=1-\Phi\left(\frac{\ln \left(t_{i}\right)-\mu}{\sigma}\right) .
\end{aligned}
$$


From equation (1), the joint likelihood function for $\mu$ and $\sigma$ given an incomplete data set is

$$
\begin{aligned}
& L\left(\mu, \sigma \mid D_{0}\right) \\
& =\left(2 \pi \sigma^{2}\right)^{-\frac{1}{2} \sum_{i=1}^{n} v_{i}} \exp \left(-\frac{1}{2 \sigma^{2}} \sum_{i=1}^{n} v_{i}\left(\ln \left(t_{i}\right)-\mu\right)^{2}\right) \\
& \quad \times \prod_{i=1}^{n} t_{i}^{-v_{i}}\left(1-\Phi\left(\frac{\log \left(t_{i}\right)-\mu}{\sigma}\right)\right)^{1-v_{i}}
\end{aligned}
$$

To construct a lognormal regression model covariates that are realized through $\mu$ are introduced by defining $\mu_{i}=\mathbf{x}_{\mathbf{i}}^{\prime} \boldsymbol{\beta}$. By defining $\tau=1 / \sigma^{2}$, the likelihood function is given can be written as

$$
\begin{aligned}
& L(\boldsymbol{\beta}, \tau \mid D) \\
& =\left(2 \pi \tau^{-1}\right)^{-\frac{1}{2} \sum_{i=1}^{n} v_{i}} \exp \left(-\frac{\tau}{2} \sum_{i=1}^{n} v_{i}\left(\ln \left(t_{i}\right)-\mathbf{x}_{\mathbf{i}}^{\prime} \boldsymbol{\beta}\right)^{2}\right) \\
& \quad \times \prod_{i=1}^{n} t_{i}^{-v_{i}}\left(1-\Phi\left(\frac{\ln \left(t_{i}\right)-\mathbf{x}_{\mathbf{i}}^{\prime} \boldsymbol{\beta}}{\tau^{-1 / 2}}\right)\right)^{1-v_{i}}
\end{aligned}
$$

A typical prior distribution for $\tau$ is a gamma prior distribution. [11] In this paper, it is supposed that $\tau \sim G\left(a_{0} / 2, b_{0} / 2\right), \quad \boldsymbol{\beta}$ has a multinormal prior distribution with $p$ vector, denoted by $N_{p}\left(\boldsymbol{\mu}_{\mathbf{0}}, \tau^{-1} \boldsymbol{\Sigma}_{\mathbf{0}}\right) .[11]$ Therefore, the posterior distribution for $\tau$ and $\boldsymbol{\beta}$ can be is

$$
\begin{aligned}
& \pi(\boldsymbol{\beta}, \tau \mid D) \propto \tau^{\frac{a_{0}+\sum_{i=1}^{n} v_{i}}{2}-1} \\
& \times \exp \left(-\frac{\tau}{2} \sum_{i=1}^{n} v_{i}\left(\ln \left(t_{i}\right)-\mathbf{x}_{\mathbf{i}}^{\prime} \boldsymbol{\beta}\right)^{2}+\left(\boldsymbol{\beta}-\boldsymbol{\mu}_{\mathbf{0}}\right)^{\prime} \boldsymbol{\Sigma}_{\mathbf{0}}^{-\mathbf{1}}\left(\boldsymbol{\beta}-\boldsymbol{\mu}_{\mathbf{0}}\right)+b_{0}\right) \\
& +\prod_{i=1}^{n} t_{i}^{-v_{i}}\left(1-\Phi\left(\tau^{1 / 2}\left(\ln \left(t_{i}\right)-\mathbf{x}_{\mathbf{i}}^{\prime} \boldsymbol{\beta}\right)\right)\right)^{1-v_{i}}
\end{aligned}
$$

\section{EXAMPLE}

This paper focuses on the wheels of the locomotive of a cargo train. While two types of locomotives with the same type of wheels are used in cargo trains, we consider only one.

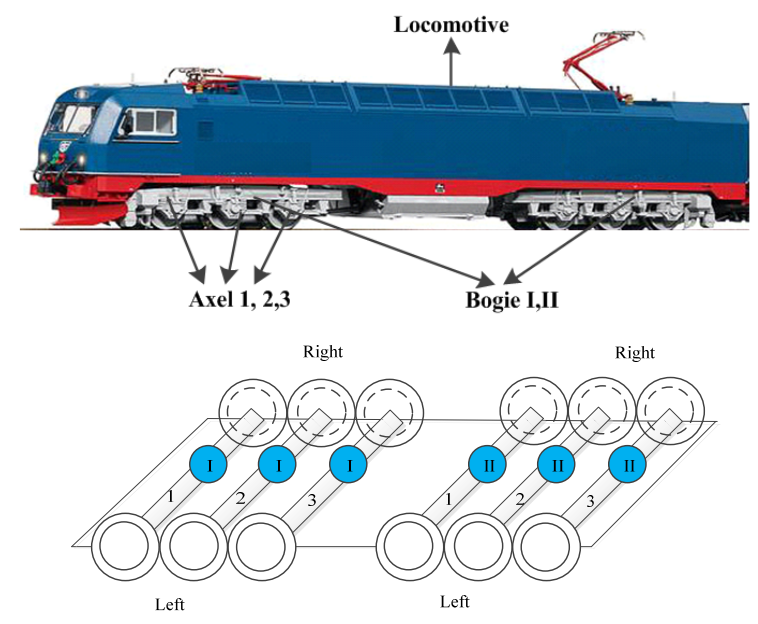

Fig. 1 Locomotive wheels' installation positions
There are two bogies for each locomotive and three axels for each bogie (Fig.1). The installed position of the wheels on a particular locomotive is specified by a bogie number (I, IInumber of bogies on the locomotive), an axel number (1, 2, 3number of axels for each bogie) and the side of the wheel on the axle (right or left) where each wheel is mounted.

The diameter of a new locomotive wheel in the studied railway company is $1250 \mathrm{~mm}$. In the company's current maintenance strategy, a wheel's diameter is measured after running a certain distance. If it is reduced to $1150 \mathrm{~mm}$, the wheel is replaced by a new one. Otherwise, it is re-profiled or other maintenance strategies are implemented. A threshold level for failure, is defined as $100 \mathrm{~mm}(=1250 \mathrm{~mm}-1150$ $\mathrm{mm}$ ). The wheel's failure condition is assumed to be reached if the diameter reaches $100 \mathrm{~mm}$.

The company's complete database also includes the diameters of all locomotive wheels at a given observation time, the total running distances corresponding to their "time to be maintained", and the wheels' bill of material (BOM) data, from which we can determine their positions.

Two assumptions are made: 1 ) for each censored datum it is supposed that the wheel is replaced; 2) degradation is linear. Only one locomotive is considered in this example to ensure that 1) all wheel's maintenance strategies are the same; 2) the axle load and running speed are obviously constant; and 3) the operational environments including routes, climates and exposure are common for all wheels.

The data set contains 46 datum points $(n=46)$ of a single locomotive throughout period November 2010 to January 2012. We take the following steps to obtain locomotive wheels' lifetime data (Fig.2):

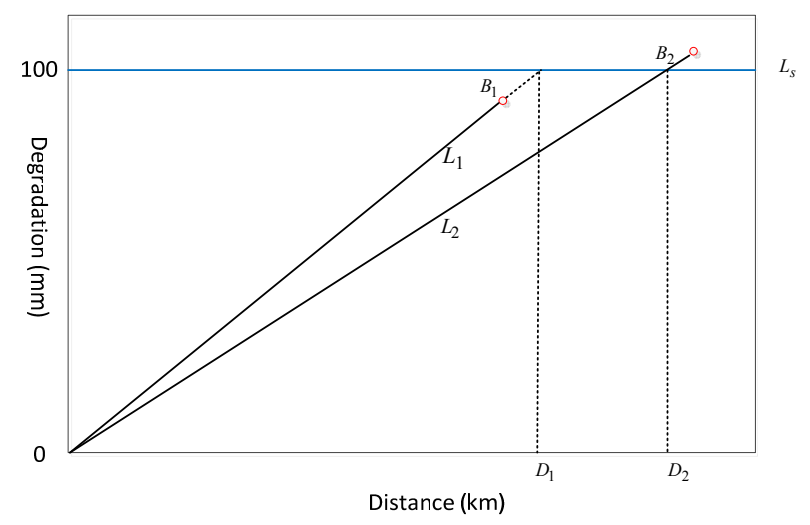

Fig.2 Plot of the wheel degradation data: one example

- Establish a threshold level $L_{S}$, where $L_{S}=100 \mathrm{~mm}(1250$ $\mathrm{mm}-1150 \mathrm{~mm}$ ).

- Transfer observed 90 records of wheel diameters at reported time $t$ to degradation data; this equals to $1250 \mathrm{~mm}$ minus the corresponding observed diameter.

- Assume a liner degradation path and construct a degradation line $L_{i}$ (e.g. $\left.L_{1}, L_{2}\right)$ using the origin point and the degradation data (e.g. $B_{1}, B_{2}$ ).

- Set $L_{s}=L_{i}$, get the point of intersection and the corresponding lifetimes data (e.g. $D_{1}, D_{2}$ ). 
For each reported datum, a wheel's installation position is documented, and as mentioned above, positioning is used in this study as a covariate. As discussed in section 3, the wheel's position (bogie, axel, and side) or covariate $\mathbf{X}$ is denoted by $x_{1}$ (bogie I: $x_{1}=1$, bogie II: $x_{1}=2$ ), $x_{2}$ (axel 1: $x_{2}=1$, axel 2: $x_{2}=2$, axel $3, x_{2}=3$ ) and $x_{3}$ (right: $x_{3}=1$, left: $x_{3}=2$ ). Correspondingly, the covariates' coefficients are represented by $\beta_{1}, \beta_{2}$, and $\beta_{3}$. In addition, $\beta_{0}$ is defined as random effect.

The calculations are implemented with the software WinBUGS[14] . A burn-in of 10,001 samples is used, with an additional 10,000 Gibbs samples for each Markov chain. Vague prior distributions are adopted here as the following: For exponential regression: $\boldsymbol{\beta} \sim N(0,0.0001)$; for Weibull regression: $\alpha \sim G(0.2,0.2), \quad \boldsymbol{\beta} \sim N(0,0.0001)$; for lognormal Regression: $\tau \sim G(1,0.01), \boldsymbol{\beta} \sim N(0,0.0001)$.

Following the convergence diagnostics (including to check Markov chains' dynamic trace, time series, GelmanRubin-Statistics, as well as to compare the MC error with Standard Deviation (SD)), [14] we consider following posterior summaries of parameters as shown in tables 1,2 and 3 for our models with censored data, including the parameters' posterior mean, standard deviation, Monte Carlo (MC) error, and 95\% HPD (highest posterior distribution density) interval. In below tables, the mean values for $\boldsymbol{\beta}$ seem quite small, that is because the measurement unit to locomotive wheels lifetime is by thousands kilometers $\left(\times 10^{3} \mathrm{~km}\right)$.

Table.1 Posteriors Summaries - Exponential Regression Model

\begin{tabular}{|c|c|c|c|c|}
\hline Parameter & Mean & SD & MC error & $95 \%$ HPD \\
\hline$\beta_{0}$ & -5.862 & 0.7355 & 0.02299 & $(-7.366,-4.452)$ \\
\hline$\beta_{1}$ & -0.07207 & 0.3005 & 0.007269 & $(-0.6672,0.5104)$ \\
\hline$\beta_{2}$ & -0.03219 & 0.1858 & 0.003797 & $(-0.3889,0.3325)$ \\
\hline$\beta_{3}$ & -0.0124 & 0.2973 & 0.00726 & $(-0.5954,0.5787)$ \\
\hline
\end{tabular}

Table.2 Posteriors Summaries - Weibull Regression Model

\begin{tabular}{|c|c|c|c|c|}
\hline Parameter & Mean & SD & MC error & $95 \%$ HPD \\
\hline$\alpha$ & 10.08 & 0.9674 & 0.05559 & $(8.234,11.76)$ \\
\hline$\beta_{0}$ & -60.47 & 5.977 & 0.3434 & $(-71.01,-49.16)$ \\
\hline$\beta_{1}$ & -0.07775 & 0.306 & 0.008339 & $(-0.6845,0.5156)$ \\
\hline$\beta_{2}$ & -0.146 & 0.2231 & 0.005801 & $(-0.5878,0.2856)$ \\
\hline$\beta_{3}$ & -0.05026 & 0.2982 & 0.007143 & $(-0.6356,0.5324)$ \\
\hline
\end{tabular}

Table.3 Posteriors Summaries - Log-normal Regression Model

\begin{tabular}{|c|c|c|c|c|}
\hline Parameter & Mean & SD & MC error & $95 \%$ HPD \\
\hline$\beta_{0}$ & 5.864 & 0.05341 & 0.001622 & $(5.76,5.97)$ \\
\hline$\beta_{1}$ & 0.06733 & 0.02174 & $5.042 \mathrm{E}-4$ & $(0.02492,0.1103)$ \\
\hline$\beta_{2}$ & 0.02077 & 0.01373 & $2.765 \mathrm{E}-4$ & $(-0.00629,0.0478)$ \\
\hline$\beta_{3}$ & 0.001102 & 0.02175 & $5.007 \mathrm{E}-4$ & $(-0.0412,0.04444)$ \\
\hline$\tau$ & 187.5 & 39.84 & 0.3067 & $(118.3,273.5)$ \\
\hline
\end{tabular}

Accordingly, the locomotive wheels' reliability functions are:

- Exponential Regression Model:

$$
\begin{aligned}
& R\left(t_{i} \mid \mathbf{X}\right) \\
& =\exp \left[-\exp \left(-5.862-0.072 x_{1}-0.032 x_{2}-0.012 x_{3}\right) \times t_{i}\right]
\end{aligned}
$$

- Weibull Regression Model:

$$
\begin{aligned}
& R\left(t_{i} \mid \mathbf{X}\right) \\
& \quad=\exp \left[-\exp \left(-60.47-0.078 x_{1}-0.146 x_{2}-0.050 x_{3}\right) \times t_{i}^{10.08}\right]
\end{aligned}
$$

- Log-normal Regression Model:

$$
\begin{aligned}
& R\left(t_{i} \mid \mathbf{X}\right) \\
& \quad=1-\Phi\left[\frac{\ln \left(t_{i}\right)-\left(5.864+0.067 x_{1}+0.02 x_{2}+0.001 x_{3}\right)}{(187.5)^{-1 / 2}}\right]
\end{aligned}
$$

Obviously, other quantities regarding lifetime distribution, including MTTF can be determined.

For model comparison, usually two main aspects are considered: the model's measure of fit and its complexity. In this paper, we adopt the Deviance Information Criterion (DIC), which utilizes the model's deviance to evaluate its measure of fit, and the effective number of parameters to evaluate its complexity. [14]

Define a Bayesian model's Bayesian deviance, denoted as $D(\theta)$, as $D(\theta)=-2 \log (p(D \mid \theta))$; Define the effective number of parameters, denoted as $p_{d}$, as:

$$
p_{d}=\bar{D}(\theta)-D(\bar{\theta})=-\int 2 \ln (p(D \mid \theta)) d \theta-(-2 \ln (p(D \mid \bar{\theta})))
$$

Then, $D I C=D(\bar{\theta})+2 p_{d}=\bar{D}(\theta)+p_{d}$. We calculate the DIC values for the above three Bayesian parametric models separately, as shown in Table 4.

Table.4 DIC Summaries

\begin{tabular}{|c|c|c|c|c|}
\hline Model & $\bar{D}(\theta)$ & $D(\bar{\theta})$ & $p_{d}$ & DIC \\
\hline Exponential & 648.98 & 645.03 & 3.95 & 652.93 \\
\hline Weibull & 472.22 & 467.39 & 4.83 & 477.05 \\
\hline Log-normal & 442.03 & 436.87 & 5.16 & 447.19 \\
\hline
\end{tabular}

Our results show that the DIC for Log-normal Regression Model is the lowest (447.19), and it is a better choice. The prediction of the locomotive wheels MTTF, following Bayesian Lognormal regression model, appears in Table.5.

It should be pointed out that the 95\% HPD interval in Bayesian Lognormal regression model for $\beta_{2}$ and $\beta_{3}$ includes 0 (Table.3). This means that, although the positioning does have an influence, in some instances, the impact on the wheel's service lifetime is not significantly strong. In our case, the bogies have more impact on service lifetime than axels or sides. Given this conclusion, we can deal with such covariates better in our future research. Besides above, other conclusions include: 1) the lifetime of the wheel installed in the second bogie is longer than that of the wheel installed in the first one; 2) the wheel installed in the third axel has a longer lifetime than that installed in the second axel, and the wheel in the second axel has a longer lifetime than the one in the first axel; 3 ) the right side wheel's lifetime is shorter than the left side. 
(Researchers from Norwegian National Rail Administration cited previously concur with this. Using condition monitoring methods on train wheels operating on the same route, they found that the wheel forces on the right and the left sides can be different, even for wheels in the same axel.). Possible causes include the influence of the earth's rotation, topographical complexity, and the position of the locomotive's centre of gravity.

Table.5 MTTF statistics based on Bayesian Lognormal Regression Model

\begin{tabular}{|c|c|c|c|c|}
\hline Bogie & Axel & Side & $\mu_{i}$ & $\begin{array}{c}\text { MTTF } \\
\left(\times 10^{3} \mathrm{~km}\right)\end{array}$ \\
\hline \multirow{6}{*}{$\begin{array}{c}\mathrm{I} \\
\left(x_{1}=1\right)\end{array}$} & \multirow{2}{*}{$\begin{array}{c}1 \\
\left(x_{2}=1\right)\end{array}$} & $\operatorname{Right}\left(x_{3}=1\right)$ & 5.9532 & 387.03 \\
\hline & & Left $\left(x_{3}=2\right)$ & 5.9543 & 387.46 \\
\hline & \multirow{2}{*}{$\begin{array}{c}2 \\
\left(x_{2}=2\right)\end{array}$} & $\operatorname{Right}\left(x_{3}=1\right)$ & 5.9740 & 395.16 \\
\hline & & Left $\left(x_{3}=2\right)$ & 5.9751 & 395.60 \\
\hline & \multirow{2}{*}{$\begin{array}{c}3 \\
\left(x_{2}=3\right)\end{array}$} & $\operatorname{Right}\left(x_{3}=1\right)$ & 5.9947 & 403.43 \\
\hline & & Left $\left(x_{3}=2\right)$ & 5.9958 & 403.87 \\
\hline \multirow{6}{*}{$\begin{array}{c}\text { II } \\
\left(x_{1}=2\right)\end{array}$} & \multirow{2}{*}{$\begin{array}{c}1 \\
\left(x_{2}=1\right)\end{array}$} & $\operatorname{Right}\left(x_{3}=1\right)$ & 6.0205 & 413.97 \\
\hline & & Left $\left(x_{3}=2\right)$ & 6.0216 & 414.43 \\
\hline & \multirow{2}{*}{$\begin{array}{c}2 \\
\left(x_{2}=2\right)\end{array}$} & $\operatorname{Right}\left(x_{3}=1\right)$ & 6.0413 & 422.67 \\
\hline & & Left $\left(x_{3}=2\right)$ & 6.0424 & 423.14 \\
\hline & \multirow{2}{*}{$\begin{array}{c}3 \\
\left(x_{2}=3\right)\end{array}$} & $\operatorname{Right}\left(x_{3}=1\right)$ & 6.0621 & 431.56 \\
\hline & & Left $\left(x_{3}=2\right)$ & 6.0632 & 432.03 \\
\hline
\end{tabular}

4 CONCLUSIONS AND FUTURE RESEARCH

This paper proposes three parametric Bayesian models for locomotive wheels' reliability analysis using degradation data: Bayesian Exponential Regression Model, Bayesian Weibull Regression Model, and Log-normal Regression Model. By introducing the covariate $\mathbf{x}_{i}$ 's linear function $\mathbf{x}_{i}^{\prime} \boldsymbol{\beta}$, these three parameter models are constructed depending on the failure rate $\lambda_{i}$ in the exponential model, the log of the rate parameter $\ln \left(\gamma_{i}\right)$ in the Weibull model and the logarithmic mean $\mu_{i}$ in the log-normal models. The proposed Bayesian survival models can deal with small and incomplete data sets and simultaneously consider the influence of several covariates. The MCMC technique via the Gibbs sampler is used here to achieve models' posteriors estimations.

The case study's results suggest that the locomotive wheels' lifetimes are different with different installed positions. In addition, the approach discussed in this paper can also be applied for analyzing cargo train wheels. The work presented also leads to the implementation of additional research:

- The assumed liner degradation path was a simple one. For more complex path models, more degradation paths need to be studied, including considering different wear rates.

- The covariates considered here are only limited to locomotive wheels' installed positions, more covariates needs to be considered later, like: temperature, applied loading, train speed, etc.
- We have chosen general prior distributions for the case study. As more information can be utilized, how to integrate different prior also need to be studied. Besides above limitations, in our later research, we also plan to consider utilization of utilization of our results to optimize maintenance strategies and related LCC (Life Cycle Cost) problems with consideration of maintenance cost.

\section{REFERENCES}

1. Braghin F, et al. A Mathematical Model to Predict Railway Wheel Profile Evolution Due to Wear. Journal of Wear. 2006. 261: 1253-1264

2. Tassini N, et al. A Numerical Model of Twin Disc Test Arrangement for the Evaluation of Railway Wheel Wear Prediction Methods. Journal of Wear. 2010. 268: 660667

3. Bernasconi A, et al. An Integrated Approach to Rolling Contact Sub-surface Fatigue assessment of Railway Wheels. Journal of Wear. 2005. 258: 973-980

4. Clayton P. Tribological Aspects of Wheel-Rail Contact: A Review of Recent Experimental Research. Journal of Wear. 1996. 191: 170-183

5. Yang C, Letourneau S. Learning to Predict Train Wheel Failures. Conference Proceedings. The 11th ACM SIGKDD International Conference on Knowledge Discovery and Data Mining (KDD 2005). Chicago, Illinois, USA.

6. Pombo J, Ambrosio J, Pereira M. A Railway Wheel Wear Prediction Tool based on A Multibody Software. Journal of Theoretical and Applied Mechanics. 2010. 48, 3:751770

7. Donato P, et al. Design and Signal Processing of A Magnetic Sensor Array for Train Wheel Detection. Journal of Senors and Actuators A. 2006. 132: 516-525

8. Stratman B, Liu Y, Mahadevan S. Structural Health Monitoring of Railroad Wheels Using Wheel Impact Load Detectors. Journal of Failure Analysis and Prevention. 2007. 7(3):218-225

9. Palo M. Condition Monitoring of Railway Vehicles: A Study on Wheel Condition for Heavy Haul Rolling Stock. Licentiate Thesis. Luleå University of Technology, Sweden. 2012

10. Freitas M A, et al. Using Degradation Data to Assess Reliability: A Case Study on Train Wheel Degradation. Journal of Quality and Reliability Engineering International. 2009, 25: 607-629

11. Ibrahim J G, Chen M H, Sinha D. Bayesian Survival Analysis. New York: Berlin Heidelberg, 2001

12. Klein J P, Moeschberger M L. Survival Analysis: Techniques for Censored and Truncated Data. SpringerVerlag New York, Inc.1997

13. Lawless. Statistical Models and Methods for Lifetime Data. John Wiley and Sons. 1982

14. Spiegelhalter D, et al. WinBUGS User Manual (Version 1.4). January, 2003. http:// www.mrc-bsu.cam.ac.uk/bugs 


\section{ACKNOWLEDGEMENTS}

The authors would like to thank Luleå Railway Research Centre (Järnvägstekniskt Centrum, Sweden) for initiating the research study and Swedish Transport Administration (Trafikverket) for providing financial support. Also we would like to thank the editor and anonymous referees for their constructive comments.

\section{BIOGRAPHIES}

Jing Lin, $\mathrm{PhD}$

Division of Operation, Maintenance and Acoustics

Luleå University of Technology

Luleå, Norrbotten 97187 Sweden

e-mail: janet.lin@ltu.se

Dr. Jing Lin is currently a researcher in the Division of Operation, Maintenance and Acoustic, at Luleå University of Technology (LTU), Sweden. She obtained her PhD degree in Management from Nanjing University of Science and Technology (NJUST), China, in April 2008; and she received the bachelor degree in Management from (NJUST) in 2003. After the college, she worked 3 years for SKF Co., Ltd as an Asset Management Consultant. Dr. Lin's research interests primarily lie in asset management and reliability. She has published 35 peer reviewed Journal and Conference papers and 1 monograph in related topics.

Matthias Asplund, $\mathrm{PhD}$ candidate

Division of Operation, Maintenance and Acoustics

Luleå University of Technology

Luleå, Norrbotten 97187 Sweden e-mail: Matthias.asplund@ltu.se

Matthias Asplund is a $\mathrm{PhD}$ student in the Division of Operation, Maintenance and Acoustic, at Luleå University of Technology (LTU), Sweden. since 2011. His research area is RAMS with railway topics in focus. He has twelve years working experience from product development, lean production, maintenance and railway engineering. He got his Master degree in Mechanical Engineering with focus on Applied Mechanics from Luleå Technical University. His last work before studies to $\mathrm{PhD}$ was Track Engineering for the Swedish Infrastructure Manager.

Aditya Parida, $\mathrm{PhD}$

Division of Operation, Maintenance and Acoustics

Luleå University of Technology

Luleå, Norrbotten 97187 Sweden

e-mail: Aditya.parida@ltu.se

Dr. Aditya Parida is an Associate Professor in the Division of Operation, Maintenance and Acoustic, at Luleå University of Technology, Sweden. He obtained his $\mathrm{PhD}$ in Operation and Maintenance Engineering. His area of research is Asset Management, Maintenance Performance Measurement and model, RCM and eMaintenance. Besides teaching, he is actively involved in research and projects. He is the author of 65 peer reviewed Journal and Conference papers, besides 3 book chapters and guest editors of four special issues of International Journals. 